Volume 8. No. 7, July 2020

International Journal of Emerging Trends in Engineering Research

Available Online at http://www.warse.org/IJETER/static/pdf/file/ijeter166872020.pdf https://doi.org/10.30534/ijeter/2020/166872020

\title{
Coupled Dynamic Thermoplasticity Problem for Transversely Isotropic Parallelepiped
}

\author{
Abduvali A. Khaldjigitov ${ }^{1}$, Mumin R. Babajanov ${ }^{2}$, Aziz A. Kalandarov ${ }^{3}$, Ravshan S. Khudazarov ${ }^{4}$ \\ ${ }^{1}$ Professor, National University of Uzbekistan, Uzbekistan, akhald@mail.ru \\ ${ }^{2}$ Faculty of Computer Engineering, Tashkent University of Information Technologies, Uzbekistan, \\ mum1975@mail.ru \\ ${ }^{3}$ Head of the Department of Information Technology, Gulistan State University, Uzbekistan, abrorshox@mail.ru \\ ${ }^{4}$ Department of Mathematics, Tashkent University of Information Technologies, Uzbekistan, \\ khudazarov@mail.ru
}

\begin{abstract}
The article frmulates a coupled thermoplastic dynamic boundary value problem based on the deformation theory of transversally isotropic bodies. The coupled boundary value problem consists of the motion equation, the thermoplasticity constitutive relations for transversely isotropic bodies, the Cauchy relation and the heat conduction equation, with the corresponding initial and boundary conditions. A coupled dynamic boundary value problem for a thermoplastic transversely isotropic parallelepiped is considered. For the numerical solution of the coupled boundary value problem, an explicit finite-difference schemes are constructed. The finite-difference equations are written in the form of recurrent relations allowing to find the components of displacement and temperature, taking into account the initial and boundary conditions at each layer in time. On the basis of the obtained numerical results, the stress-strain state of a transversely isotropic thermoplastic parallelepiped is investigated and the propagation of the plastic zone is shown.
\end{abstract}

Key words: Coupled problem, thermoplasticity, temperature, displacements, difference scheme, plastic zone, deformation, stress.

\section{INTRODUCTION}

Investigation the joint influence of the thermomechanical forces on the strength and durability of structures is an actual problem of engineering. Usually this type of problems in the framework of the thermoelacticity or thermoplasticity can be described by the system of motion and heat conduction equations with a corresponding initial and thermo-mechanical boundary conditions.

The coupled thermoelastic boundary value problems was investigated firstly by Biot [1]. In the works of Lord \& Shulman[2] has been introduced a generalized coupled theory with a wave-type heat equation The following works [3-10] are devoted to the development of the theoretical foundations of coupled thermomechanical deformations of solids subject to both large and inelastic deformations. The coupled thermoplasticity problems are considered in $[7,9-11,15,16]$. The coupled and uncoupled thermomechanical boundary value problems are numerically solved in following works [12-14, 17-18,20].

The paper [18] considers the statement and numerical solution of partially related problems of thermoelasticity. In work [19] the analysis of heat transfer of water heating convectors is given.

In Sections $\sim 2$ using the deformation thermoplasticity theory for transversely isotropic materials the dynamic coupled boundary value problem are formulated. Note, the boundary value problems, are reduced to the system consisting of three nonlinear motion and one heat partial differential equations.

In Section 3, using the finite difference method, the discreet analogy of the coupled dynamic boundary value problems based on deformation theory of thermoplasticity are constructed. The finite-difference equations resolved in relative to the desired quantities are reduced to recurrent formulas and taking into account the initial and boundary conditions a numerical results are determined.

In section 4, a clamped on all surfaces parallelepiped with a given initial sinusoidal thermal load is considered. The described process using the deformation theory of thermoplasticity is formulated as a coupled boundary value problem and numerically solved. The temperature distribution and the appearing of the plasticity zones inside the parallelepiped under the given initial and boundary conditions are investigated.

\section{FORMULATION OF THE COUPLED DYNAMIC THERMOPLASTICITY BOUNDARY VALUE PROBLEM}

Consider a coupled dynamic boundary value problem of thermoplasticity for a transversely isotropic parallelepiped consisting of the motion equation

$$
\sum_{j=1}^{3} \frac{\partial \sigma_{i j}}{\partial x_{j}}+X_{i}=\rho \frac{\partial^{2} u_{i}}{\partial t^{2}}, \quad i=1,2,3
$$

constitutive relation of the deformation theory of thermoplasticity for transversely isotropic bodies $[17,20]$ 
Abduvali A. Khaldjigitov et al., International Journal of Emerging Trends in Engineering Research, 8(7), July 2020, 3958 - 3964

$$
\sigma_{i j}=\left\{\begin{array}{l}
C_{i j k e} \varepsilon_{k l}-\beta_{i j}\left(T-T_{0}\right) \delta_{i j}, \text { if } \quad p<p^{*}, q<q^{*} \\
C_{i j k e} \varepsilon_{k l}-\beta_{i j}\left(T-T_{0}\right) \delta_{i j}-2\left(\lambda_{2}-\lambda_{2}^{\prime}\right)\left(1-\frac{p^{*}}{p}\right) p_{i j}- \\
-2\left(\lambda_{5}-\lambda_{5}^{\prime}\right)\left(1-\frac{q^{*}}{q}\right) q_{i j}, \text { if } p \geq p^{*}, q \geq q^{*}
\end{array}\right.
$$

Cauchy relations

$$
\varepsilon_{i j}=\frac{1}{2}\left(\frac{\partial u_{i}}{\partial x_{j}}+\frac{\partial u_{j}}{\partial x_{i}}\right)
$$

heat conduction equations for anisotropic bodies

$$
\lambda_{i j} T_{i j}-c_{\varepsilon} \dot{T}-T \cdot \beta_{i j} \cdot \dot{\varepsilon}_{i j}=0
$$

with initial

$$
\left.u_{i}\right|_{t=t_{0}}=\varphi_{i},\left.\quad \dot{u}_{i}\right|_{t=t_{0}}=\psi_{i},\left.\quad T\right|_{t=t_{0}}=T_{0}
$$

and boundary conditions

$$
\left.u_{i}\right|_{\Sigma_{1}}=u_{i}^{0},\left.\quad T\right|_{\Sigma}=\bar{T}_{0},\left.\quad \sum_{j=1}^{3} \sigma_{i j} n_{j}\right|_{\Sigma_{2}}=S_{i}
$$

where, $\sigma_{i j}$ - stress tensor, $\varepsilon_{i j}$ - strain tensor, $u_{i}$ displacement components, $X_{i}$ - volume force, $C_{i j k l}$ - tensor of the fourth rank determining the mechanical properties of a material, $T$-temperature, $T_{0}$-initial temperature, $p, q$ - strain tensor intensity, $p^{*}, q^{*}-$ elastic limits in the longitudinal and transverse directions of a transversely isotropic body, $\beta_{i j}-$ corresponds to thermal expansion coefficients, $\rho$-density of the body, $\delta_{i j}$ - delta Kronecker symbol, $n_{j}$ external normal to the surface $\Sigma_{2}, S_{1}, S_{2}, S_{3}$ - components of the external load vector, $\lambda_{i j}-$ is the heat flow coefficients, $c_{\varepsilon}$-denotes heat coefficient at a constant deformation, $\Sigma=\Sigma_{1}+\Sigma_{2}-$ the surface of the body under consideration, the upper point denotes the time derivative.

Equations (1)-(6) represent a dynamic coupled boundary value problem of thermoplasticity for transversely isotropic bodies. The boundary value problem (1)-(6), after some transformations, can be written in the following form, with respect to displacements and temperature

$$
\begin{aligned}
& C_{1111} \frac{\partial^{2} u}{\partial x^{2}}+C_{1212} \frac{\partial^{2} u}{\partial y^{2}}+C_{1313} \frac{\partial^{2} u}{\partial z^{2}}+\left(C_{1122}+C_{1212}\right) \frac{\partial^{2} v}{\partial x \partial y}+ \\
& +\left(C_{1133}+C_{1313}\right) \frac{\partial^{2} w}{\partial x \partial z}-\beta_{11} \frac{\partial T}{\partial x}-F_{1}=\rho \\
& C_{1212} \frac{\partial^{2} v}{\partial x^{2}}+C_{2222} \frac{\partial^{2} v}{\partial y^{2}}+C_{2223} \frac{\partial^{2} v}{\partial z^{2}}+\left(C_{2211}+C_{1212}\right) \frac{\partial^{2} u}{\partial x \partial y}+ \\
& +\left(C_{2233}+C_{22323}\right) \frac{\partial^{2} w}{\partial y \partial z}-\beta_{22} \frac{\partial T}{\partial y}-F_{2}=\rho \frac{\partial^{2} v}{\partial t^{2}}
\end{aligned}
$$

$$
\begin{aligned}
& C_{1313} \frac{\partial^{2} w}{\partial x^{2}}+C_{2323} \frac{\partial^{2} w}{\partial y^{2}}+C_{3333} \frac{\partial^{2} w}{\partial z^{2}}+\left(C_{3311}+C_{1313}\right) \frac{\partial^{2} u}{\partial x \partial z}+ \\
& +\left(C_{3322}+C_{2323}\right) \frac{\partial^{2} v}{\partial y \partial z}-\beta_{33} \frac{\partial T}{\partial z}-F_{3}=\rho \frac{\partial^{2} w}{\partial t^{2}}
\end{aligned}
$$

heat flow equation

$$
\begin{aligned}
& \lambda_{11} \frac{\partial^{2} T}{\partial x^{2}}+\lambda_{22} \frac{\partial^{2} T}{\partial y^{2}}+\lambda_{33} \frac{\partial^{2} T}{\partial z^{2}}-c_{\varepsilon} \frac{\partial T}{\partial t}- \\
& -T\left(\beta_{11} \frac{\partial^{2} u}{\partial x \partial t}+\beta_{22} \frac{\partial^{2} v}{\partial y \partial t}+\beta_{33} \frac{\partial^{2} w}{\partial z \partial t}\right)=0
\end{aligned}
$$

with corresponding initial

$$
\begin{gathered}
\left.u(x, y, z, t)\right|_{t=0}=\varphi_{1},\left.v(x, y, z, t)\right|_{t=0}=\varphi_{2}, w(x, y, z, t) \\
\left.\frac{\partial u}{\partial t}\right|_{t=0}=\psi_{1},\left.\quad \frac{\partial v}{\partial t}\right|_{t=0}=\psi_{2},\left.\frac{\partial w}{\partial t}\right|_{t=0}=\psi_{3}, \\
\left.T(x, y, z, t)\right|_{t=0}=T_{0}
\end{gathered}
$$

and boundary conditions with respect to displacements

$$
\left.u(x, y, z, t)\right|_{\Sigma_{1}}=u^{0},\left.v(x, y, z, t)\right|_{\Sigma_{1}}=v^{0},\left.w(x, y, z, t)\right|_{\Sigma_{1}}=w^{0}
$$

or loads

$$
\begin{aligned}
& \left.\left(\sigma_{11} n_{1}+\sigma_{12} n_{2}+\sigma_{13} n_{3}\right)\right|_{\Sigma_{2}}=S_{1},\left.\quad\left(\sigma_{21} n_{1}+\sigma_{22} n_{2}+\sigma_{23} n_{3}\right)\right|_{\Sigma_{2}}=S_{2}, \\
& \left.\left(\sigma_{31} n_{1}+\sigma_{32} n_{2}+\sigma_{33} n_{3}\right)\right|_{\Sigma_{2}}=S_{3}
\end{aligned}
$$

and temperatures

$$
\left.T(x, y, z, t)\right|_{\Sigma}=\bar{T}_{0}
$$

where

$$
\begin{aligned}
& F_{1}=2\left(\lambda_{2}-\lambda_{2}^{\prime}\right)\left(1-\frac{p^{*}}{p}\right)\left[\frac{\partial\left(\varepsilon_{11}-\varepsilon_{22}\right)}{2 \partial x_{1}}+\frac{\partial \varepsilon_{12}}{\partial x_{2}}\right]+2\left(\lambda_{5}-\lambda_{5}^{\prime}\right)\left(1-\frac{p^{*}}{p}\right) \frac{\partial \varepsilon_{13}}{\partial x_{3}}, \\
& F_{2}=2\left(\lambda_{2}-\lambda_{2}^{\prime}\right)\left(1-\frac{p^{*}}{p}\right)\left[\frac{\partial \varepsilon_{21}}{\partial x_{2}}+\frac{\partial\left(\varepsilon_{22}-\varepsilon_{11}\right)}{2 \partial x_{2}}\right]+2\left(\lambda_{5}-\lambda_{5}^{\prime}\right)\left(1-\frac{p^{*}}{p}\right) \frac{\partial \varepsilon_{23}}{\partial x_{3}}, \\
& F_{3}=2\left(\lambda_{5}-\lambda_{5}^{\prime}\right)\left(1-\frac{q^{*}}{q}\right)\left[\frac{\partial \varepsilon_{31}}{\partial x_{1}}+\frac{\partial \varepsilon_{32}}{\partial x_{2}}\right] .
\end{aligned}
$$

if $p \geq p^{*}, q \geq q^{*}$.

\section{THE FINITE DIFFERENCE EQUATIONS FOR COUPLED DYNAMIC BOUNDARY VALUE PROBLEMS OF THERMO PLASTISITY}

The coupled thermoplasticity boundary value problem based on deformation theory of thermoplasticity for transversely isotropic materials presents by the eqs. (7-10) considered in the domain $\Omega=\left\{t \geq 0,0 \leq x \leq l_{1}, 0 \leq y \leq l_{2}, 0 \leq z \leq l_{3}\right\}$. In order to construct a grid equations consider three sets of parallel lines $x_{i}=i h_{1}\left(i=\overline{0, N_{1}}\right), y_{j}=j h_{2}\left(j=\overline{0, N_{2}}\right)$, $z_{k}=k h_{3}\left(\mathrm{k}=\overline{0, N_{3}}\right) \quad$ where $\quad h_{1}=l_{1} / N_{1}, \quad h_{2}=l_{2} / N_{2}$, $h_{3}=l_{3} / N_{3} \quad$, in the domain $\Omega$ and taking $t_{m}=m \tau \quad(\mathrm{m}=0,1,2, \ldots)$, where $\tau$ - a step on the axis $t$. Then replacing the derivatives in eqs.(7-10) by difference quotients, we obtain 
Abduvali A. Khaldjigitov et al., International Journal of Emerging Trends in Engineering Research, 8(7), July 2020, 3958 - 3964

$$
\begin{aligned}
& C_{1111} \frac{u_{i+1, j, k}^{n}-2 u_{i, j, k}^{n}+u_{i-1, j, k}^{n}}{h_{1}^{2}}+C_{1212} \frac{u_{i, j+1, k}^{n}-2 u_{i, j, k}^{n}+u_{i, j-1, k}^{n}}{h_{2}^{2}}+ \\
& +C_{1313} \frac{u_{i, j k+1}^{n}-2 u_{i, j, k}^{n}+u_{i, j, k-1}^{n}}{h_{3}^{2}}+\left(C_{1122}+C_{1212}\right) \frac{v_{i+1, j+1, k}^{n}-v_{i-1, j+1, k}^{n}-v_{i+1, j-1, k}^{n}+v_{i-1, j-1, k}^{n}}{4 h h_{2}}+ \\
& +\left(C_{1333}+C_{1313} \frac{u_{i+1, j, k+1}^{n}-u_{i-1, j, k+1}^{n}-u_{i+1, j, k-1}^{n}+u_{i-1, j, k-1}^{n}}{4 h_{1} h_{3}}-\right. \\
& -\beta_{11} \frac{T_{i+1, j, k}^{n}-T_{i-1, j, k}^{n}}{2 h_{1}}-F_{1}=\rho \frac{u_{i, j, k}^{n+1}-2 u_{i, j, k}^{n}+u_{i, j, k}^{n-1}}{\tau^{2}}
\end{aligned}
$$

$$
\begin{aligned}
& C_{1212} \frac{v_{i+1, j, k}^{n}-2 v_{i, j, k}^{n}+v_{i-1, j, k}^{n}}{h_{1}^{2}}+C_{222} \frac{v_{i, j+1, k}^{n}-2 v_{i, j, k}^{n}+v_{i, j-1, k}^{n}}{h_{2}^{2}}+ \\
& +C_{2232} \frac{v_{i, j, k+1}^{n}-2 v_{i, j, k}^{n}+v_{i, j, k-1}^{n}}{h_{3}^{2}}+\left(C_{2211}+C_{1212}\right) \frac{u_{i+1, j+1, k}^{n}-u_{i-1, j+1, k}^{n}-u_{i+1, j-1, k}^{n}+u_{i-1, j-1, k}^{n}}{4 h_{1} h_{2}}+ \\
& \left(C_{2233}+C_{2223}\right) \frac{w_{i, j+1, k+1}^{n}-w_{i, j-1, k+1}^{n}-w_{i, j+1, k-1}^{n}+w_{i, j-1, k-1}^{n}}{4 h_{2} h_{3}}- \\
& -\beta_{22} \frac{T_{i, j+1, k}^{n}-T_{i, j-1, k}^{n}}{2 h_{2}}-F_{2}=\rho \frac{v_{i, j, k}^{n+1}-2 v_{i, j, k}^{n}+v_{i, j, k}^{n-1}}{\tau^{2}}
\end{aligned}
$$$$
C_{1313} \frac{w_{i+1, j, k}^{n}-2 w_{i, j, k}^{n}+w_{i-1, j, k}^{n}}{h_{1}^{2}}+C_{2233} \frac{w_{i, j+1, k}^{n}-2 w_{i, j, k}^{n}+w_{i, j-1, k}^{n}}{h_{2}^{2}}+
$$$$
+C_{3333} \frac{w_{i, j, k+1}^{n}-2 w_{i, j, k}^{n}+w_{i, j, k-1}^{n}}{h_{3}^{2}}+\left(C_{3311}+C_{1313} \frac{u_{i+1, j, k+1}^{n}-u_{i-1, j, k+1}^{n}-u_{i+1, j, k-1}^{n}+u_{i-1, j, k-1}^{n}}{4 h_{1} h_{3}}+\right.
$$$$
+\left(C_{3322}+C_{2223}\right) \frac{v_{i, j+1, k+1}^{n}-v_{i, j-1, k+1}^{n}-v_{i, j+1, k-1}^{n}+v_{i, j-1, k-1}^{n}}{4 h_{1} h_{2}}-
$$$$
-\beta_{33} \frac{T_{i, j, k+1}^{n}-T_{i, j, k-1}^{n}}{2 h_{3}}-F_{3}=\rho \frac{w_{i, j, k}^{n+1}-2 w_{i, j, k}^{n}+w_{i, j, k}^{n-1}}{\tau^{2}}
$$$$
\lambda_{11} \frac{T_{i+1, j, k}^{n}-2 T_{i, j, k}^{n}+T_{i-1, j, k}^{n}}{h_{1}^{2}}+\lambda_{2} \frac{T_{i, j+1, k}^{n}-2 T_{i, j, k}^{n}+T_{i, j-1, k}^{n}}{h_{2}^{2}}+\lambda_{33} \frac{T_{i, j, k+1}^{n}-2 T_{i, j, k}^{n}+T_{i, j, k-1}^{n}}{h_{3}^{2}}-
$$$$
-c_{\varepsilon} \frac{T_{i, j, k}^{n+1}-T_{i, j, k}^{n}}{\tau}-T_{i j}\left(\beta_{11} \frac{u_{i+1, j, k}^{n+1}-u_{i-1, j k}^{n+1}-u_{i+1, j, k}^{n-1}+u_{i-1, j, k}^{n-1}}{4 h_{1} \tau}+\right.
$$$$
\left.+\beta_{22} \frac{v_{i, j+1, k}^{n+1}-v_{i, j-1, k}^{n+1}-v_{i, j+1, k}^{n-1}+v_{i, j, 1, k}^{n-1}}{4 h_{2} \tau}+\beta_{33} \frac{w_{\frac{1+i, k+1}{n+1}-1}-w_{i, j, k-1}^{n+1}-w_{i, j, k+1}^{n-1}+w_{i, j, k-1}^{n-1}}{4 h_{3} \tau}\right)=0
$$

Solving the difference equations (11-14), with respect to $u_{i, j, k}^{n+1}, v_{i, j, k}^{n+1}, w_{i, j, k}^{n+1}$ and $T_{i, j, k}^{n+1}$, accordingly, we obtain

$$
\begin{aligned}
& u_{i, j, k}^{n+1}=\frac{\tau^{2}}{\rho}\left(C_{1111} \frac{u_{i+1, j, k}^{n}-2 u_{i, j, k}^{n}+u_{i-1, j, k}^{n}}{h_{1}^{2}}+C_{1212} \frac{u_{i, j 1, k}^{n}-2 u_{i, j, k}^{n}+u_{i, j-1, k}^{n}}{h_{2}^{2}}+\right. \\
& +C_{1313} \frac{u_{i, j, k+1}^{n}-2 u_{i, j, k}^{n}+u_{i, j, k-1}^{n}}{h_{3}^{2}}+\left(C_{1122}+C_{1212}\right) \frac{v_{i+1, j+1, k}^{n}-v_{i-1, j+1, k}^{n}-v_{i+1, j-1, k}^{n}+v_{i-1, j-1, k}^{n}}{4 h_{1} h_{2}}+ \\
& +\left(C_{1333}+C_{1313} \frac{u_{i+1, j, k+1}^{n}-w_{i-1, j, k+1}^{n}-u_{i+1, j, k-1}^{n}+u_{i-1, j, k-1}^{n}}{4 h_{1} h_{3}}-\right. \\
& \left.-\beta_{11} \frac{T_{i+1, j, k}^{n}-T_{i-1, j, k}^{n}}{2 h_{1}}-F_{1}\right)+2 u_{i, j, k}^{n}-u_{i, j, k}^{n-1}
\end{aligned}
$$

$$
\begin{aligned}
& v_{i, j, k}^{n+1}=\frac{\tau^{2}}{\rho}\left(C_{1212} \frac{v_{i+1, j, k}^{n}-2 v_{i, j, k}^{n}+v_{i-1, j, k}^{n}}{h_{1}^{2}}+C_{222} \frac{v_{i, j+1, k}^{n}-2 v_{i, j, k}^{n}+v_{i, j-1, k}^{n}}{h_{2}^{2}}+\right. \\
& +C_{2223} \frac{v_{i, j, k+1}^{n}-2 v_{i, j, k}^{n}+v_{i, j, k-1}^{n}}{h_{3}^{2}}+\left(C_{2211}+C_{1212}\right) \frac{u_{i+1, j+1, k}^{n}-u_{i-1, j+1, k}^{n}-u_{i+1, j-1, k}^{n}+u_{i-1, j-1, k}^{n}}{4 h_{1} h_{2}}+ \\
& +\left(C_{223}+C_{2233}\right) \frac{w_{i, j+1, k+1}^{n}-w_{i, j-1, k+1}^{n}-w_{i, j 1, k-1}^{n}+w_{i, j-1, k-1}^{n}}{4 h_{2} h_{3}}- \\
& \left.-\beta_{22} \frac{T_{i, j+1, k}^{n}-T_{i, j-1, k}^{n}}{2 h_{2}}-F_{2}\right)+2 v_{i, j, k}^{n}-v_{i, j, k}^{n-1} \\
& w_{i, j k}^{n+1}=\frac{\tau^{2}}{\rho}\left(C_{1313} \frac{w_{i+1, j k}^{n}-2 w_{i, j, k}^{n}+w_{i-1, j, k}^{n}}{h_{1}^{2}}+C_{2233} \frac{w_{i, j+1, k}^{n}-2 w_{i, j, k}^{n}+w_{i, j-1, k}^{n}}{h_{2}^{2}}+\right. \\
& +C_{3333} \frac{w_{i, j, k+1}^{n}-2 w_{i, j k}^{n}+w_{i, j, k-1}^{n}}{h_{3}^{2}}+\left(C_{3311}+C_{1313} \frac{u_{i+1, j, k+1}^{n}-u_{i-1, j k+1}^{n}-u_{i+1, j, k-1}^{n}+u_{i-1, j, k-1}^{n}}{4 h h_{3}}+\right. \\
& +\left(C_{332}+C_{2233}\right) \frac{v_{i, j+1, k+1}^{n}-v_{i, j 1, k+1}^{n}-v_{i, j+1, k-1}^{n}+v_{i, j 1, k-1}^{n}}{4 h_{1} h_{2}}- \\
& \left.-\beta_{33} \frac{T_{i, j, k+1}^{n}-T_{i, j, k-1}^{n}}{2 h_{3}}-F_{3}\right)+2 w_{i, j, k}^{n}-w_{i, j, k}^{n-1} \\
& T_{i, j, k}^{n+1}=\frac{\tau}{c_{\varepsilon}}\left(\lambda_{11} \frac{T_{i+1, j, k}^{n}-2 T_{i, j, k}^{n}+T_{i-1, j, k}^{n}}{h_{1}^{2}}+\lambda_{22} \frac{T_{i, j+1, k}^{n}-2 T_{i, j, k}^{n}+T_{i, j-1, k}^{n}}{h_{2}^{2}}+\right. \\
& +\lambda_{33} \frac{T_{i, j, k+1}^{n}-2 T_{i, j, k}^{n}+T_{i, j, k-1}^{n}}{h_{3}^{2}}-T_{i j}\left(\beta_{11} \frac{u_{i+1, j, k}^{n+1}-u_{i-1, j, k}^{n+1}-u_{i+1, j, k}^{n-1}+u_{i-1, j, k}^{n-1}}{4 h_{1} \tau}+\right. \\
& \left.\left.+\beta_{22} \frac{v_{i, j+1, k}^{n+1}-v_{i, j, 1, k}^{n+1}-v_{i, j+1, k}^{n-1}+v_{i, j-1, k}^{n-1}}{4 h_{2} \tau}+\beta_{33} \frac{w_{i, j, k+1}^{n+1}-v_{i, j, k-1}^{n+1}-w_{i, j, k+1}^{n-1}+w_{i, j, k-1}^{n-1}}{4 h_{3} \tau}\right)\right)+T_{i, j, k}^{n}
\end{aligned}
$$

The resulting recurrent formulas (15-18), taking into account the initial and boundary conditions, make it possible to find the numerical values of the sought functions at each layer in time. First, we solve the thermoelastic problem; in this case, the values of $F_{1}, F_{2}, F_{3}$ in formulas (15)-(17) are trivial. On each layer, plastic zones are determined in time, where the values of the sought functions are re-calculated.

\section{NUMERICAL TEST}

Let's consider a clamped all sides parallelepiped with an internal initial sinusoidal temperature at the time $t=0$. The described process using the deformation thermoplasticity theory has been modelled by the eqs. (7-10). The initial conditions take the following forms:

$$
\begin{gathered}
\left.u(x, y, z, t)\right|_{t=0}=0,\left.v(x, y, z, t)\right|_{t=0}=0,\left.w(x, y, z, t)\right|_{t=0}=0 \\
\left.\frac{\partial u}{\partial t}\right|_{t=0}=0,\left.\quad \frac{\partial v}{\partial t}\right|_{t=0}=0,\left.\quad \frac{\partial w}{\partial t}\right|_{t=0}=0 \\
\left.T(x, y, z, t)\right|_{t=0}=T_{0} \sin \left(\pi x_{i}\right) \sin \left(\pi y_{j}\right) \sin \left(\pi z_{k}\right)
\end{gathered}
$$

and boundary conditions

$$
\begin{gathered}
\left.u(x, y, z, t)\right|_{\Sigma}=0,\left.v(x, y, z, t)\right|_{\Sigma}=0,\left.w(x, y, z, t)\right|_{\Sigma}=0 \\
\left.T(x, y, z, t)\right|_{\Sigma}=0 .
\end{gathered}
$$


Elastic moduli, hardening moduli, elastic limits, heat capacity at constant deformation, thermal expansion tensor, thermal conductivity tensor, density had the following dimensionless values:

$\mathrm{C}_{1122}=0.21, \mathrm{C}_{1133}=0.19, \mathrm{C}_{2233}=0.19, \mathrm{C}_{3333}=5.356$,

$\mathrm{C}_{2323}=2.39, \mathrm{C}_{1313}=2.39$

$\mathrm{C}_{1212}=2.735, \mathrm{C}_{1111}=5,68, \mathrm{C}_{2222}=5,68, \mathrm{~b} 11=0.25, \mathrm{~b} 22=\mathrm{b} 11$, b33 $=0.37$,

$\lambda_{11}=0.03, \quad \lambda_{22}=0.03, \quad \lambda_{33}=0.01, \lambda_{2}^{\prime}=2.5, \quad \lambda_{5}^{\prime}=2.24, \rho=0.81$,

$c_{\varepsilon}=3.5, \lambda_{2}^{\prime}=2.5, \quad \lambda_{5}^{\prime}=2.24, \quad \tau=0.01$,

$p^{*}=0.08, q^{*}=0.04, T_{0}=20, \mathrm{~N}_{1}=\mathrm{N}_{2}=\mathrm{N}_{3}=10$.

Below we give the zones of plasticity in different layers in time and in sections along the coordinate axes of the considered transversely isotropic parallelepiped.

Table 1: Strain tensor intensity values $p$, at $z=0,7, t=0,07$

\begin{tabular}{|c|c|c|c|c|c|c|}
\hline $\begin{array}{c}\mathrm{x} \\
\mathrm{y}\end{array}$ & 0 & 0.1 & 0.2 & 0.3 & 0.4 & 0.5 \\
\hline 0 & 0 & 0.00000 & 0.00000 & 0.00000 & 0.00000 & 0.00000 \\
\hline 0.1 & 0 & 0.10273 & 0.11462 & 0.12015 & 0.11984 & 0.11940 \\
\hline 0.2 & 0 & 0.11439 & 0.08931 & 0.07044 & 0.04453 & 0.02822 \\
\hline 0.3 & 0 & 0.12001 & 0.07069 & 0.05097 & 0.02713 & 0.00359 \\
\hline 0.4 & 0 & 0.11963 & 0.04476 & 0.02715 & 0.01420 & 0.00012 \\
\hline 0.5 & 0 & 0.11914 & 0.02836 & 0.00372 & 0.00027 & 0.00008 \\
\hline 0.6 & 0 & 0.11960 & 0.04472 & 0.02715 & 0.01420 & 0.00012 \\
\hline 0.7 & 0 & 0.11995 & 0.07066 & 0.05097 & 0.02713 & 0.00359 \\
\hline 0.8 & 0 & 0.11432 & 0.08935 & 0.07055 & 0.04465 & 0.02822 \\
\hline 0.9 & 0 & 0.10289 & 0.11478 & 0.12024 & 0.11987 & 0.11940 \\
\hline 1 & 0 & 0.00000 & 0.00000 & 0.00000 & 0.00000 & 0.00000 \\
\hline
\end{tabular}

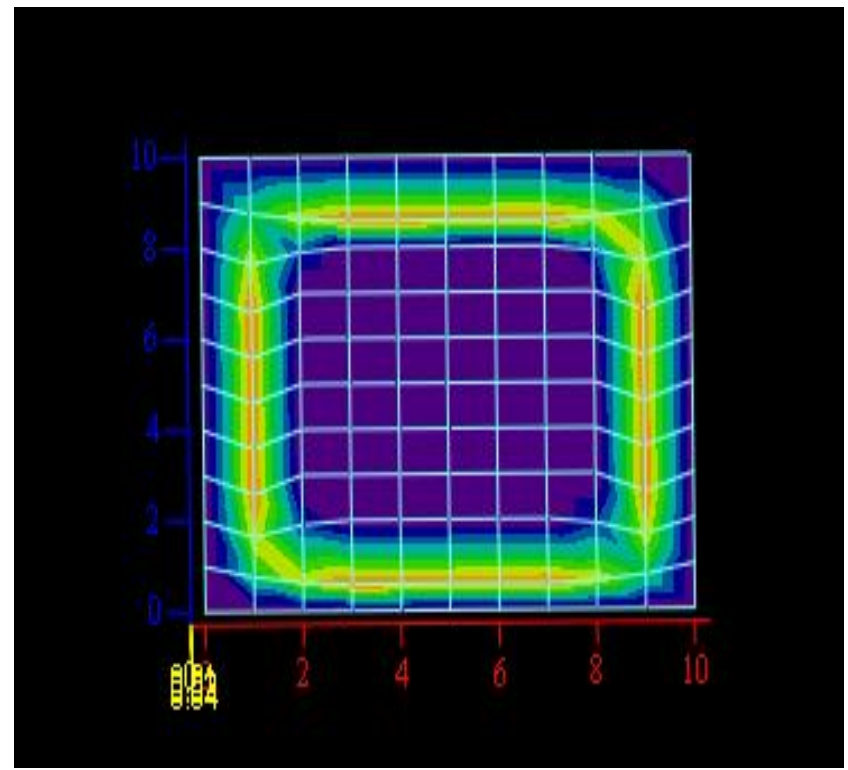

Figure 1: Plasticity zone by intensity tensor of deformations $p$ in the $X O Y$ plane at $z=0.7, t=0.07\left(p \geq p^{*}\right), p^{*}=0.08$
Table 2: Strain tensor intensity values $q$, at $y=0.9, t=0.07$

\begin{tabular}{|c|c|c|c|c|c|c|}
\hline $\begin{array}{c}\mathrm{x} \\
\mathrm{z}\end{array}$ & 0 & 0.1 & 0.2 & 0.3 & 0.4 & 0.5 \\
\hline 0 & 0 & 0.00000 & 0.00000 & 0.00000 & 0.00000 & 0.00000 \\
\hline 0.1 & 0 & 0.05295 & 0.07524 & 0.09512 & 0.10844 & 0.11311 \\
\hline 0.2 & 0 & 0.04995 & 0.07114 & 0.09020 & 0.10297 & 0.10744 \\
\hline 0.3 & 0 & 0.03817 & 0.05432 & 0.06887 & 0.07861 & 0.08203 \\
\hline 0.4 & 0 & 0.02026 & 0.02880 & 0.03651 & 0.04166 & 0.04346 \\
\hline 0.5 & 0 & 0.00000 & 0.00000 & 0.00000 & 0.00000 & 0.00000 \\
\hline 0.6 & 0 & 0.02026 & 0.02880 & 0.03651 & 0.04166 & 0.04346 \\
\hline 0.7 & 0 & 0.03817 & 0.05432 & 0.06887 & 0.07861 & 0.08203 \\
\hline 0.8 & 0 & 0.04995 & 0.07114 & 0.09020 & 0.10297 & 0.10744 \\
\hline 0.9 & 0 & 0.05295 & 0.07524 & 0.09512 & 0.10844 & 0.11311 \\
\hline 1 & 0 & 0.00000 & 0.00000 & 0.00000 & 0.00000 & 0.00000 \\
\hline
\end{tabular}

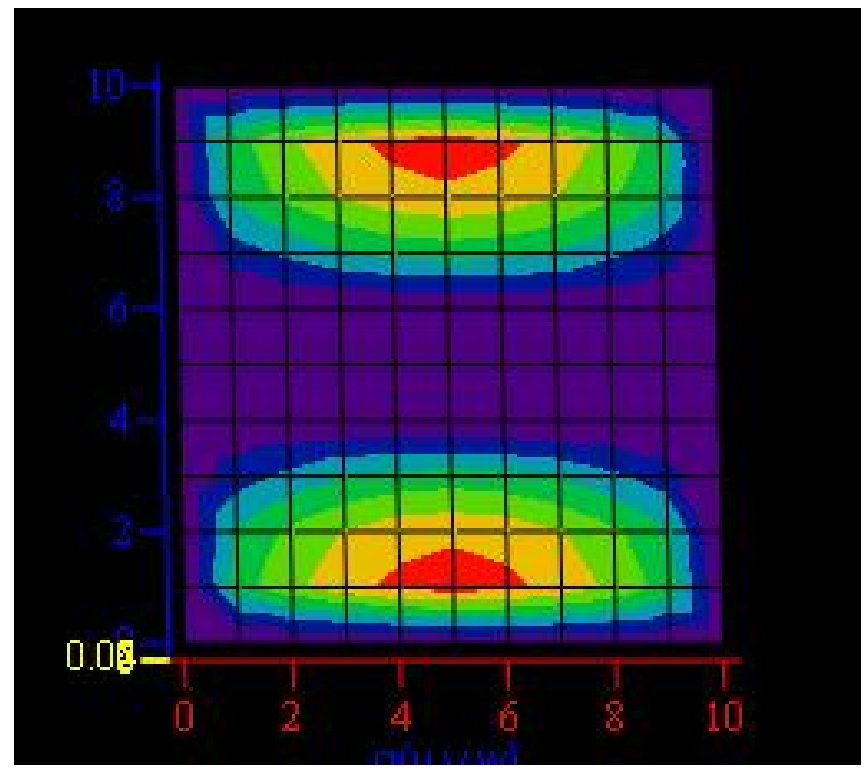

Figure 2: Plasticity zone by intensity of tensor deformations $q$ in the XOY plane at $z=0.8, t=0.07\left(q \geq q^{*}\right), q^{*}=0.04$

Next, we present the numerical results and 3D graphs of the functions $u(x, y, z, t), v(x, y, z, t), w(x, y, z, t)$ and $T(x, y, z, t)$ in the area under consideration.

Table 3: Displacement values $u(x, y, z, t)$ at $z=0.3$ and $t=0.09$

\begin{tabular}{|c|c|c|c|c|c|c|}
\hline $\begin{array}{c}\mathrm{x} \\
\mathrm{y}\end{array}$ & 0 & 0.1 & 0.2 & 0.3 & 0.4 & \multicolumn{1}{|c|}{0.5} \\
\hline 0 & 0 & 0.00000 & 0.00000 & 0.00000 & 0.00000 & 0.00000 \\
\hline 0.1 & 0 & -0.01143 & -0.01372 & -0.01076 & -0.00574 & 0.0000 \\
\hline 0.2 & 0 & -0.01898 & -0.02335 & -0.01833 & -0.00976 & 0.00000 \\
\hline 0.3 & 0 & -0.02558 & -0.03158 & -0.02482 & -0.01322 & 0.00000 \\
\hline 0.4 & 0 & -0.03002 & -0.03706 & -0.02913 & -0.01551 & 0.00000 \\
\hline 0.5 & 0 & -0.03158 & -0.03896 & -0.03062 & -0.01631 & 0.00000 \\
\hline 0.6 & 0 & -0.03005 & -0.03706 & -0.02913 & -0.01551 & 0.00000 \\
\hline 0.7 & 0 & -0.02562 & -0.03159 & -0.02482 & -0.01322 & 0.00000 \\
\hline 0.8 & 0 & -0.01903 & -0.02335 & -0.01833 & -0.00976 & 0.00000 \\
\hline 0.9 & 0 & -0.01147 & -0.01372 & -0.01072 & -0.00569 & 0.00000 \\
\hline 1 & 0 & 0.00000 & 0.00000 & 0.00000 & 0.00000 & 0.00000 \\
\hline
\end{tabular}




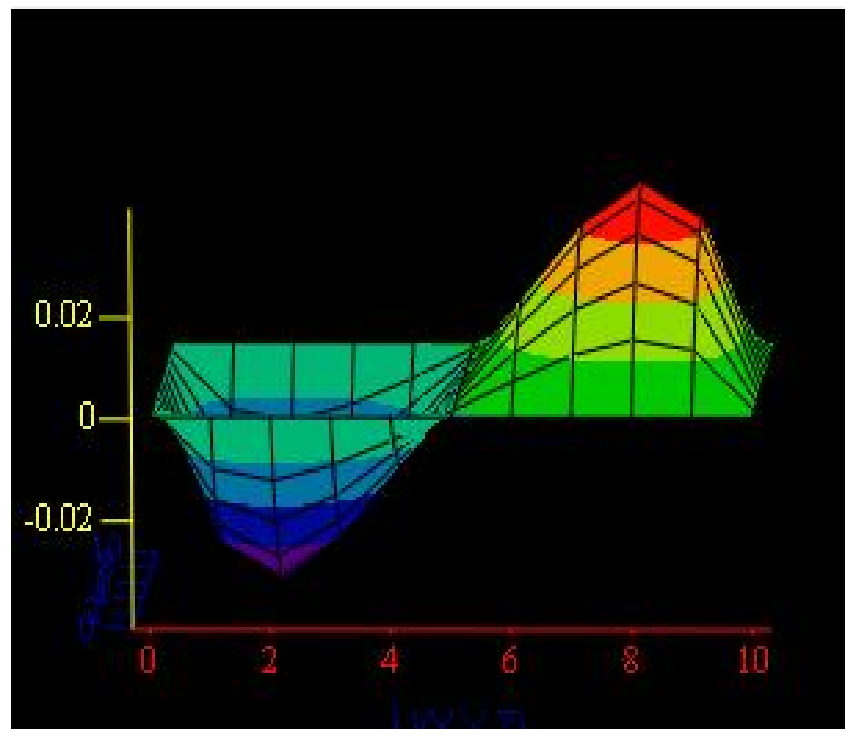

Figure 3: The graph of the distribution of the function $u(x, y, z, t)$ in the plane $X O Y$ at $z=0.3$ and $t=0.09$

Table 4: Displacement values $v(x, y, z, t)$ at $z=0.3$ and $t=0.09$

\begin{tabular}{|c|c|c|c|c|c|c|}
\hline $\begin{array}{c}\mathrm{x} \\
\mathrm{y}\end{array}$ & 0 & 0.1 & 0.2 & 0.3 & 0.4 & 0.5 \\
\hline 0 & 0 & 0.00000 & 0.00000 & 0.00000 & 0.00000 & 0.00000 \\
\hline 0.1 & 0 & -0.01135 & -0.01903 & -0.02568 & -0.03013 & 0.00000 \\
\hline 0.2 & 0 & -0.01362 & -0.02337 & -0.03165 & -0.03713 & 0.00000 \\
\hline 0.3 & 0 & -0.01066 & -0.01834 & -0.02486 & -0.02918 & 0.00000 \\
\hline 0.4 & 0 & -0.00567 & -0.00977 & -0.01324 & -0.01554 & 0.00000 \\
\hline 0.5 & 0 & -0.00000 & 0.00000 & 0.00000 & 0.00000 & 0.00000 \\
\hline 0.6 & 0 & 0.00567 & 0.00977 & 0.01324 & 0.01554 & 0.00000 \\
\hline 0.7 & 0 & 0.01066 & 0.01834 & 0.02486 & 0.02918 & 0.00000 \\
\hline 0.8 & 0 & 0.01362 & 0.02337 & 0.03165 & 0.03713 & 0.00000 \\
\hline 0.9 & 0 & 0.01135 & 0.01903 & 0.02568 & 0.03013 & 0.00000 \\
\hline 1 & 0 & 0.00000 & 0.00000 & 0.00000 & 0.00000 & 0.00000 \\
\hline
\end{tabular}

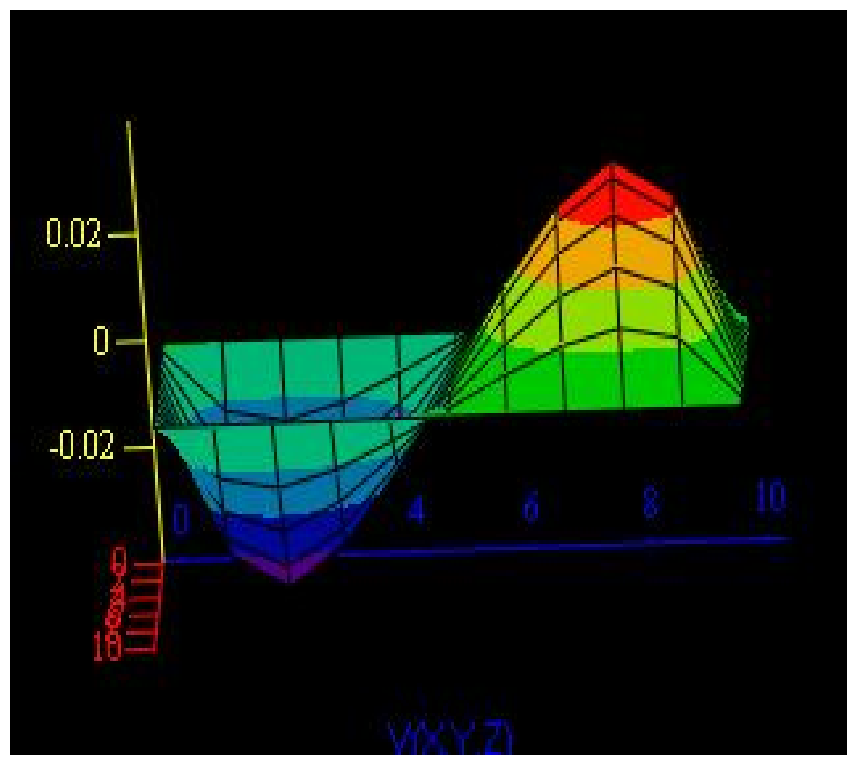

Figure 4: The graph of the distribution of the function $v(x, y, z, t)$ in the plane $X O Y$ at $z=0.3$ and $t=0.09$
Table 5: Displacement values $w(x, y, z, t)$ at $\mathrm{x}=0.3$ and $t=0.09$

\begin{tabular}{|c|c|c|c|c|c|c|}
\hline $\mathrm{X}$ & 0 & 0.1 & 0.2 & 0.3 & 0.4 & 0.5 \\
\hline 0 & 0 & 0.00000 & 0.00000 & 0.00000 & 0.00000 & 0.00000 \\
\hline 0.1 & 0 & -0.01695 & -0.02967 & -0.04034 & -0.04735 & -0.04978 \\
\hline 0.2 & 0 & -0.01983 & -0.03530 & -0.04812 & -0.05651 & -0.05941 \\
\hline 0.3 & 0 & -0.01558 & -0.02775 & -0.03785 & -0.04445 & -0.04673 \\
\hline 0.4 & 0 & -0.00831 & -0.01481 & -0.02019 & -0.02372 & -0.02493 \\
\hline 0.5 & 0 & 0.00000 & 0.00000 & 0.00000 & 0.00000 & 0.00000 \\
\hline 0.6 & 0 & 0.00831 & 0.01481 & 0.02019 & 0.02372 & 0.02493 \\
\hline 0.7 & 0 & 0.01558 & 0.02775 & 0.03785 & 0.04445 & 0.04673 \\
\hline 0.8 & 0 & 0.01983 & 0.03530 & 0.04812 & 0.05651 & 0.05941 \\
\hline 0.9 & 0 & 0.01695 & 0.02967 & 0.04034 & 0.04735 & 0.04978 \\
\hline 1 & 0 & 0.00000 & 0.00000 & 0.00000 & 0.00000 & 0.00000 \\
\hline
\end{tabular}

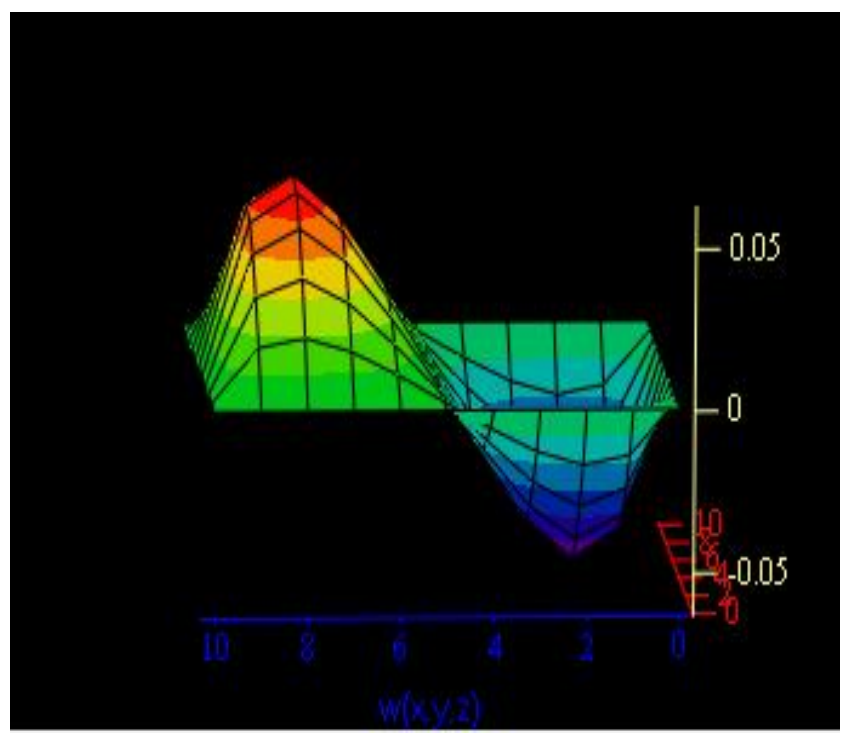

Figure 5: The graph of the distribution of the function $w(x, y, z, t)$ in the plane $Z O Y$ at $\mathrm{x}=0.3$ and $t=0.09$

Table 6: Temperature values $T(x, y, z, t)$ at $z=0.5$ and $t=0.05$

\begin{tabular}{|c|c|l|l|l|l|l|}
\hline $\mathrm{x}$ & 0 & \multicolumn{1}{|c|}{0.1} & \multicolumn{1}{|c|}{0.2} & \multicolumn{1}{|c|}{0.3} & 0.4 & 0.5 \\
\hline 0 & 0 & 0.00000 & 0.00000 & 0.00000 & 0.00000 & 0.00000 \\
\hline 0.1 & 0 & 1.98896 & 3.63207 & 4.98561 & 5.86017 & 6.16171 \\
\hline 0.2 & 0 & 3.63200 & 6.63959 & 9.11426 & 10.71307 & 11.26434 \\
\hline 0.3 & 0 & 4.98561 & 9.11433 & 12.51135 & 14.70607 & 15.46281 \\
\hline 0.4 & 0 & 5.86019 & 10.71313 & 14.70607 & 17.28578 & 18.17527 \\
\hline 0.5 & 0 & 6.16175 & 11.26439 & 15.46281 & 18.17527 & 19.11052 \\
\hline 0.6 & 0 & 5.86019 & 10.71310 & 14.70607 & 17.28578 & 18.17527 \\
\hline 0.7 & 0 & 4.98561 & 9.11427 & 12.51135 & 14.70607 & 15.46281 \\
\hline 0.8 & 0 & 3.63200 & 6.63949 & 9.11426 & 10.71307 & 11.26434 \\
\hline 0.9 & 0 & 1.98893 & 3.63200 & 4.98556 & 5.86015 & 6.16171 \\
\hline 1 & 0 & 0.00000 & 0.00000 & 0.00000 & 0.00000 & 0.00000 \\
\hline
\end{tabular}




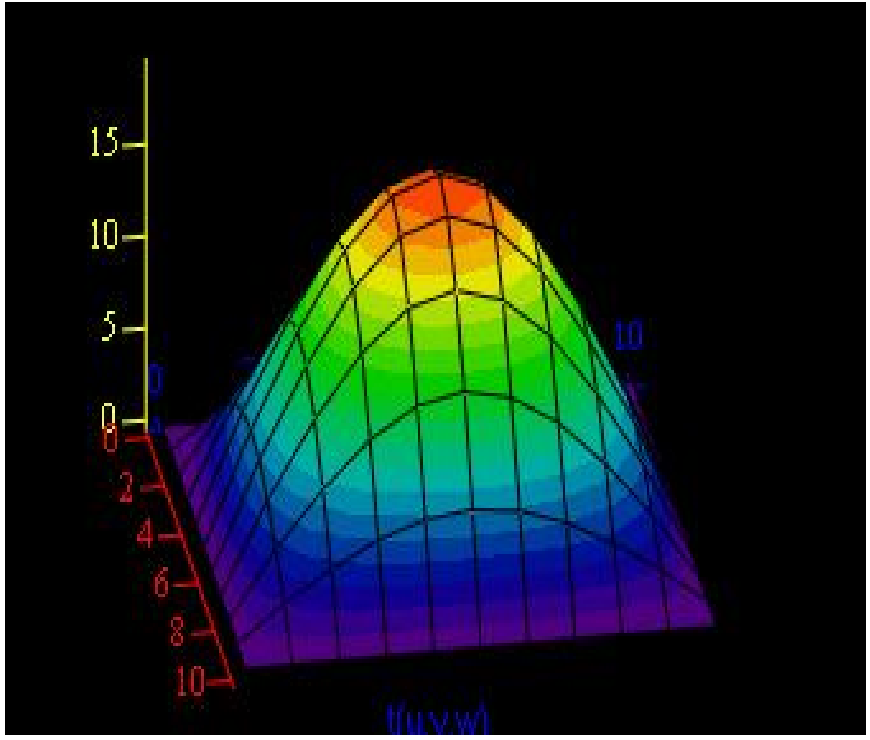

Figure 6: Temperature distribution graph of $T(x, y, z, t)$ in the plane $X O Y$ at $z=0.5$ and $t=0.05$

Note that, in the considered model problem, the axis of rotation was the OZ axis. Tables (1-2) and Figures (1-2) show the appearance of plastic zones, and Table 6 and Figure 6 show the temperature field. Since the initial and boundary conditions are set symmetrically, in Tables (3-5) and Figures (3-5) you can see the symmetry of the displacements $u(x, y, z)$ and $v(x, y, z)$, and displacements $w(x, y, z)$ are different from others. This shows the effect of anisotropy on the elastic-plastic state of a transversely isotropic parallelepiped and the reliability of the numerical results obtained.

\section{CONCLUSION}

A coupled dynamic thermoplastic boundary value problem for the deformation theory of transversely isotropic bodies is formulated. A discrete analogue of the problem is compiled by the finite difference method. On the basis of explicit finite-difference equations, recurrent formulas are obtained that allow calculating the numerical values of the required functions. A numerically dynamic coupled first boundary value problem on a thermoplastic transversely isotropic parallelepiped is solved. 3D-graphs of the distribution of displacement functions $u(x, y, z), v(x, y, z), w(x, y, z)$ and temperatures $T(x, y, z)$. The propagation of plasticity zones in various sections of a transversely isotropic parallelepiped under the action of a temperature field is investigated.

\section{REFERENCES}

1. M. Biot. Thermo elasticity and irreversible thermo-dynamics. J. Appl. Phys., 27, pp.240-253. 1956. https://doi.org/10.1063/1.1722351

2. H.W. Lord and Y. Shulman. A generalized dynamical theory of thermoplasticity. J. Mech. Phys. Solids, 1967, vol.15, pp.299-309

3. H. M. Youssef and A.S. Al-Felali. Generalized thermo elasticity Problem of Material Subjected to Thermal
Loading Due to Laser Pulse. Applied Mathematics, 2012, 3, pp. 142-146.

4. I.K. Senchenkov and N.F. Andrushko. Thermal Effects in a PhysicallyNonlinear Cylinder under Impulsive Loading // Int. Appl. Mech. 2005.41, N 8. pp. 890-894. https://doi.org/10.1007/s10778-005-0156-7

5. J. Aboudi. The effective thermomechanical behavior of inelastic fiber-reinforced materials // Int. J. Eng. sci. -1985.- Vol. 23, -No 7. pp.773-787.

6. R.M. Jones. Deformation theory of plasticity. Bull Ridge Corporation, Virginia, 2009, 622 p.

7. Z. Sloderbach. Generalized coupled thermoplasticity taking into account large strains: Part I. Conditions of uniqueness of the solution of boundary-value problem and bifurcation criteria. Math. and Mech. of solids.2010, 15, 308-327.

8. D. Helm. Stress computation in finite thermoplasticity. Int. J. Plasticity, 22, pp.1699-1727, 2006

9. J.C. Simo and C. Miehe. Associative coupled thermoplasticity at finite strains: Formulation, numerical analysis and implementation. Computer Methods in Applied Mechanics and Engineering 98 (1), pp.41-104

10. Z. Sloderbach. Generalized coupled thermoplasticity taking into account large strains: Part I. Conditions of uniqueness of the solution of boundary-value problem and bifurcation criteria. Math. and Mech. of solids.2010, 15, pp. 308-327.

11. A.A. Khaldjigitov, A.A. Qalandarov, NikM.A.Asri Long and Z. Eshquvatov. Numerical solution of $1 D$ and 2D thermoplastic coupled problems. International journal of modern physics, 2012, Vol. 9, pp. 503-510. https://doi.org/10.1142/S2010194512005594

12. A.A. Kalandarov and M.R. Babadjanov. Numerical simulation of the coupled dynamic thermoplastics problem for orthotropic bodies. International journal of computer science and Mobile computing, vol. 8 Issue 9, September 2019, pp. 182-189.

13. A.A. Qalandarov and A.A. Khaldjigitov. Mathematical and numerical modeling of the coupled dynamic thermo elastic problem for isotropic bodies. TWMS Journal of Pure and Applied Mathematics. Vol. 11, N. 1, 2020, pp. 119-126.

14. A.M. Pulatov, A.M. Ikramov and A.A. Khaldjigitov. Computer Modeling of Elastoplastic Stress state of Fibrous Composites with hole//Coupled Systems Mechanics, An International Journal, Techno-Press Ltd., Yuseong-gu Daejeon, Korea, 2019, Vol.8, №4, pp. 299-313.

15. A.A. Khaldjigitov, Nik M.A. Asri Long., A. Qalandarov and Z. Eshquvatov. Mathematical and numerical modelling of the thermoplastic coupled problem. International conference on mathematical sciences and statistics 2013. Singapore, Springer, 2014, pp. 69-75. https://doi.org/10.1007/978-981-4585-33-0_8

16. A.A. Khaldjigitov, Y.S. Yusupov, R.S. Khudazarov and D.A. Sadullaeva. On the thermoplasticity constitutive relations for isotropic and transversely isotropic materials. International Journal of Mechanical and 
Production Engineering Research and Development (IJMPERD), 2019, Vol.9, Issue 4, pp. 467-478.

17. A.A. Khaldjigitov, A.A. Kalandarov and Y.S. Yusupov. Coupled problems of thermoelasticity and thermoplasticity. Fan and technology press, Tashkent, 2019, 193 p.(in Russian)

18. A.A. Kalandarov, A. Kalandarov, S. Allayarov, S. Kulmamatov, S. Islikov and A. Adilov. Numerical modeling of partially coupled problems of thermoelasticity. International Journal of Advanced Trends in Computer Science and Engineering. Vol. 9, №3, 2020. pp. 3095-3099.

https://doi.org/10.30534/ijatcse/2020/92932020

19. S.M. Usikov. Simulation of heat transfer by water heating convectors. International Journal of Emerging Trends in Engineering Research. Vol. 8, №3, 2020. pp. 726-734.

https://doi.org/10.30534/ijeter/2020/19832020

20. A.A. Khaldjigitov, R.S. Khudazarov and D.A. Sagdullaeva The theory of plasticity and thermoplasticity for anisotropic materials. Fan and technology press. Tashkent, 2015, 315 p.(in Russian) 